\title{
The Effect Analysis of Carrier Gas Flow Rate on the Rapid Gas Chromatographic Separation System
}

\author{
Xu Zhang $\mathbb{D}^{1}{ }^{1}$ Sixiang Zhang, ${ }^{2}$ Wei Zhou, ${ }^{2}$ and Yang Qi ${ }^{1}$ \\ ${ }^{1}$ Tianjin Key Laboratory of High Speed Cutting and Precision Machining, School of Mechanical Engineering, Tianjin University of \\ Technology and Education, Tianjin, China 300222 \\ ${ }^{2}$ School of Mechanical Engineering, Hebei University of Technology, Tianjin, China 300100
}

Correspondence should be addressed to Xu Zhang; 52914262@qq.com

Received 8 April 2020; Revised 25 September 2020; Accepted 23 October 2020; Published 7 November 2020

Academic Editor: Liwei Shi

Copyright (c) $2020 \mathrm{Xu}$ Zhang et al. This is an open access article distributed under the Creative Commons Attribution License, which permits unrestricted use, distribution, and reproduction in any medium, provided the original work is properly cited.

Odor pollution did not only disturb the human normal life but also aroused the attention of environmental researchers and environmental protection departments. Therefore, the research on odor detecting method and instrument is important to theory and application. On this basis, the self-developed microfluidic chip capillary column is used in our odor detecting system. In this paper, lead the chip column into the chromatography separation system, with its small size, high efficiency, easy integration, and other characteristics to replace the original traditional column. The chip column was used in many gas experiments for several typical VOCs. At different carrier gas flow rates, the baseline value, toluene response, and toluene and methyl sulfide mixed gas separation were compared to verify the experiment to determine the optimal carrier gas flow rate in accordance with its response and separation degree. Under the premise of ensuring column efficiency as high as possible, it is determined that the optimal carrier gas flow rate is $6 \mathrm{ml} / \mathrm{min}$. This paper shows the most proper carrier gas flow rate of our odor detecting system with the self-developed microfluidic chip capillary column.

\section{Introduction}

In recent years, industrial and agricultural production processes and household waste have emitted a large amount of volatile organic compounds (VOCs), causing a series of atmospheric pollution problems. VOCs cause harm to the human respiratory system, digestive system, and endocrine system. Common psychological and emotional injuries include irritability, dizziness, nausea, and vomiting. Environmental pollution has attracted the attention of all countries in the world. The environmental protection monitoring department not only wants to know where the pollution source is but also wants to know the type and concentration of pollutants. At present, the gas detection method at home and abroad is mainly gas chromatography. Chromatography technology is a general term for the separation of complex samples and is an important method for modern separation detection and analysis [1]. The traditional gas separation and detection equipment has a large volume and mass, the operation process is complex and time-consuming, the analysis speed is slow, and the separation gas mixture uses a longer column, resulting in a long analysis cycle. The research group developed and manufactured a chip-type column for this purpose. It has features such as small sample size, high detection efficiency, low use cost, portability, ease of integration with other technical equipment, and good compatibility [2]. The gas chromatography separation and detection system involved in this article uses Beijing Minnick's ten-way valve as the sample injection system, the chip-type column as the separation system, and the PID (Photoionization Detector) as the detection system. According to the rate theory, the carrier gas flow rate affects the column. Selecting the optimal carrier gas flow rate is of great significance for the evaluation of the instrument's performance and detection accuracy. 


\section{Experimental Part}

2.1. Separation Principle of the Chip Column. The chip column uses the principle of separation by gas chromatography. In gas chromatography, the sample gas to be analyzed is rapidly carried by the carrier gas into the column. The column contains a solid or liquid stationary phase. The polarity, boiling point, or the physical and chemical properties of the adsorbed sample are different. As a result, the components are constantly distributed or adsorbed/desorbed between the stationary phase and fluidity, resulting in different rates of the components flowing out of the column, so that the retention time of the components in the column is different, in chronological order flow out of the column and into the end of the detector for detection [3].

2.2. Processing of the Chip Column. The chip column utilizes the fine sandblasting jet process to etch the surface of the borosilicate glass and adopts an anodic bonding processing method to process a Pyrex type borosilicate glass material chip with a size of $115 \mathrm{~mm} \times 60 \mathrm{~mm} \times 6 \mathrm{~mm}$ as shown in Figure 1 . The channel is designed as an S-channel with a circular cross section, which has the advantage of ensuring that the gas molecules of the components travel the same path inside and outside the chip column channel with the same column volume [4]. The channel has an inner diameter of $400 \mu \mathrm{m}$ and a length of $5.8 \mathrm{~m}$. In order to achieve the separation of VOCs, three kinds of fixing solutions, 100\% dimethylpolysiloxane, $14 \%$ cyanopropylphenyl-86\% dimethylpolysiloxane, and polyethylene glycol, were, respectively, applied. The pipe connection method of the chip is connected by means of a clamp fixture and is connected by a PTFE pipe with a threaded connection, and the connection head is connected by a conical pressure ring and can withstand a temperature of $300^{\circ} \mathrm{C}$.

\section{Chromatographic Rate Theory}

3.1. Several Parameters for Evaluating Column Performance. The theoretical plate number $N$ and the theoretical plate height $H$ are the two main indicators reflecting the column performance of the column [5]. The calculation formula of $N$ is

$$
N=5.54\left(\frac{t_{R}}{W_{1 / 2}}\right)^{2}=16\left(\frac{t_{R}}{W_{b}}\right)^{2},
$$

where $t_{R}$ is the retention time, which refers to the time from the start of sampling until the sample passes the column to produce the highest peak; $W_{b}$ is the width of the bottom of the peak, which refers to the tangent line at the inflection point of the chromatographic peak outflow curve. The distance between the base of the peak or the base line intersecting two points, $W_{1 / 2}$, is the half width of the peak, which refers to the distance between the line parallel to the base of the peak at the midpoint of the peak height and the point of intersection on both sides of the peak [6].

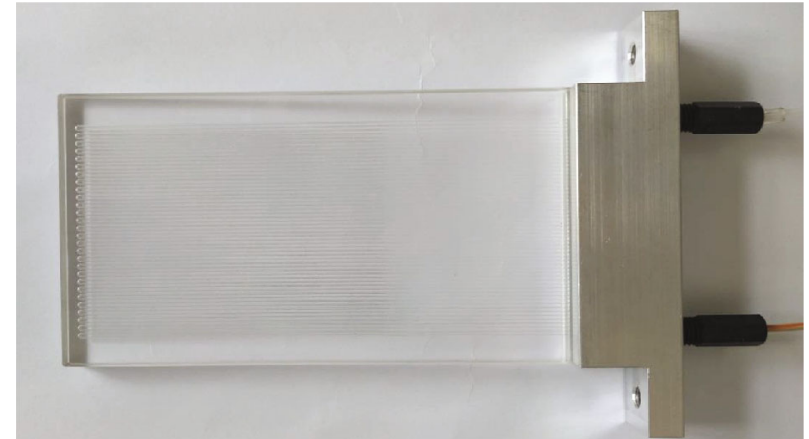

FIGURE 1: The self-developed microfluidic chip capillary column.

$H$ 's formula is

$$
H=\frac{L}{N},
$$

where $L$ is the length of the column.

It can be seen that the narrowing of the chromatographic peaks and the height of the trays will increase the column efficiency. $N$ and $H$ are the main indicators that describe the performance of a column.

The degree of separation of components-the degree of separation [7] is calculated as

$$
R=\frac{2\left(t_{R 2}-t_{R 1}\right)}{Y_{1}+Y_{2}},
$$

where $Y_{1}, Y_{2}$ is the peak width of the peak.

The size of $R$ can quantitatively reflect the degree of separation between components. When the chromatographic peak shape is asymmetrical or there is a slight overlap between adjacent two peaks, it is difficult to measure the peak width at the bottom. Instead, half width can be used instead. At this time, the resolution $R$ can be expressed as

$$
R=\frac{1.18\left(t_{R 2}-t_{R 1}\right)}{Y_{1 / 2(1)}+Y_{1 / 2(2)}},
$$

where $Y_{1 / 2(1)}, Y_{1 / 2(2)}$ is the peak width at the half height of the peak.

3.2. Rate Theory. The column efficiency is the highest when the column and the sample are at a certain flow rate [8], and the relationship of $H-u$ is

$$
H=A+\frac{B}{u}+C u
$$

where $H$ is the tray height, $u$ is the carrier gas velocity, and $A, B$, and $C$ are three constants, where $A$ is the eddy diffusion term, $B$ is the molecular diffusion coefficient, and $C$ is the mass transfer resistance factor. Equation (5) is a simplified version of the Van Deemter equation. According to equation (5), the height $H$ of the tray measured at different flow rates is plotted against the flow rate $u$ to obtain the $H-u$ 


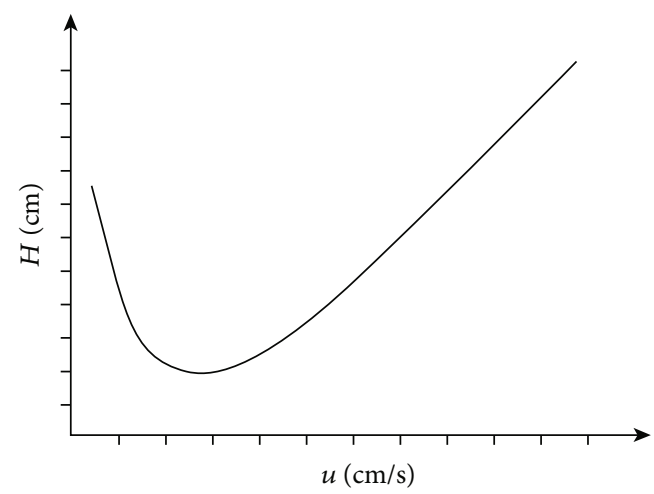

Figure 2: Relation curve between plate height and the carrier gas line speed.

curve, as shown in Figure 2. At the lowest point of the $H-u$ curve, the height of the theoretical plate $H$ is the smallest, and the efficiency of the column is the highest. The flow rate corresponding to this point is the best flow rate $u_{\text {best }}$. $H_{\text {min }}$ and $u_{\text {best }}$ can be differentiated by equation (5). Seek, that is,

$$
\begin{gathered}
\frac{d H}{d u}=-\frac{B}{u^{2}}+C=0, \\
u_{\text {best }}=\sqrt{\frac{B}{C} .}
\end{gathered}
$$

Bring formula (6) into formula (5):

$$
H_{\min }=A+2 \sqrt{B C} .
$$

In actual work, try to shorten the analysis time without significantly affecting the efficiency of the column. The flow rate is usually slightly higher than the optimal flow rate.

The relationship of the constant terms $A, B$, and $C$ is brought into equation (5).

$$
H=2 \lambda d_{p}+\frac{2 \gamma D_{g}}{\mu}+\left[\frac{0.01 k^{2}}{(1+k)^{2}} \cdot \frac{d_{p}^{2}}{D_{g}}+\frac{2}{3} \cdot \frac{k}{(1+k)^{2}} \cdot \frac{d_{f}^{2}}{D_{1}}\right] \mu .
$$

According to equation (9), the column packing uniformity, carrier particle size, carrier gas type, carrier gas flow rate, column temperature, and liquid film thickness of the stationary phase have effects on column efficiency and peak expansion.

\section{Experiments and Data}

4.1. Experimental Device and Conditions. In order to understand the effect of carrier gas flow on separation of mixtures, the self-developed odor detecting online system is introduced in these experiments. The system is shown in Figure 3.

There are six units in the system, including the gas channel unit, injection unit, separating unit, detecting unit, controlling unit, and data processing unit. This system has

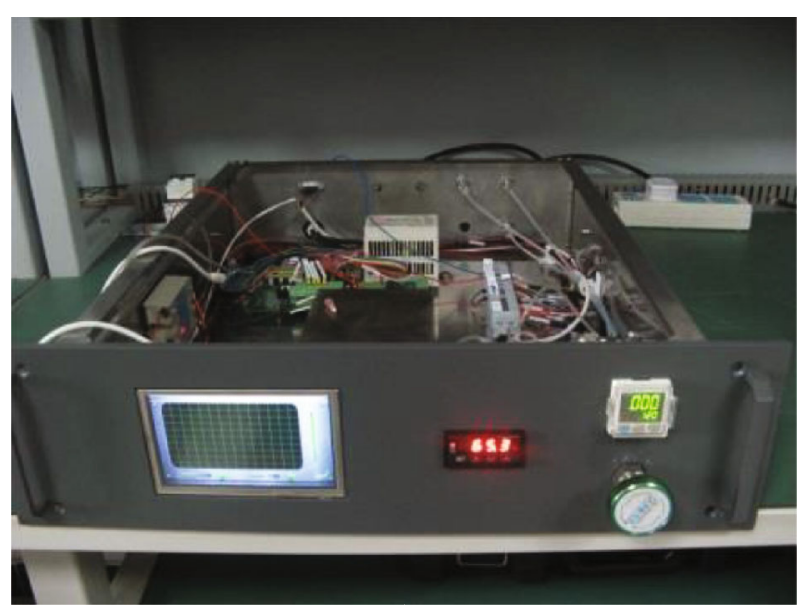

Figure 3: Self-developed odor detecting online system.

passed the stability testing, systematic linearity testing, and sensitivity testing. The testing experiments show that the baseline fluctuation value is less than $2.3 \mathrm{mV}$; the linearity of the system for xylene, ethanol, and butane is $99.98 \%$, $99.99 \%$, and $99.98 \%$, respectively. The sensitivity was $1 \mathrm{ppm}, 0.8 \mathrm{ppm}$, and $0.6 \mathrm{ppm}$, respectively [9]. We will not elaborate the testing experiments of our self-developed odor detecting online system.

The following experiment is done under the normal operating conditions. The normal operating conditions are as follows: (1) $99.999 \%$ nitrogen $\left(\mathrm{N}_{2}\right)$ was used as carrier gas; (2) the lab environmental temperature is at $25^{\circ} \mathrm{C}$; (3) the pressure in the laboratory is $0.101 \mathrm{MPa}$.

4.2. Effect of Carrier Gas Flow on Baseline Value. Baseline refers to the signal curve produced by the detector when only nitrogen passes through the column and detector under normal operating conditions. The ideal stable baseline should be a horizontal straight line. The peak height is the vertical distance between the highest point of the chromatographic peak and the bottom of the peak and the vertical distance between the highest point of the chromatographic peak and the baseline. In gas chromatography, the peak height is one of the commonly used quantitative parameters. From the theory of velocity, the flow affects the height of the theoretical plate, thus affecting the size of the baseline value. By changing the flow rate before the column, the voltage value of the PID is monitored with a voltmeter. After the baseline is stabilized, the corresponding baseline value is obtained. We used 99.999\% nitrogen $\left(\mathrm{N}_{2}\right)$ as carrier gas in this experiment. The carrier gas flow rate changed from $0 \mathrm{ml} / \mathrm{min}$ to $9 \mathrm{ml} / \mathrm{min}$ at $25^{\circ} \mathrm{C}$ in the lab. The effect of carrier gas flow on baseline values is shown in Figure 4. It shows that the baseline value was low while the carrier gas flow speeded up.

4.3. Effect of Carrier Gas Flow Rate on Toluene Response. From the rate theory, the flow rate of the carrier gas has an opposite effect on the mass transfer resistance term and the vertical diffusion term. If the carrier gas velocity $u$ increases, the longitudinal diffusion term decreases so that the column 


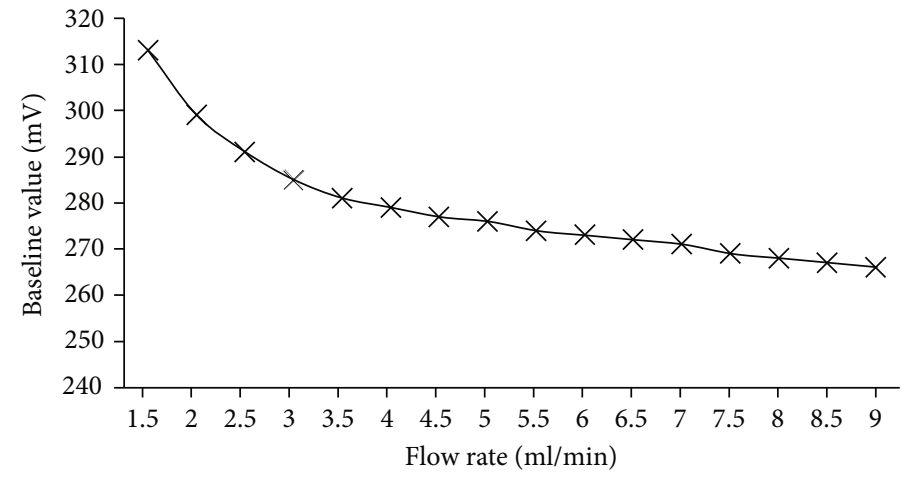

FigURE 4: Effect of carrier gas flow rate on the baseline value.

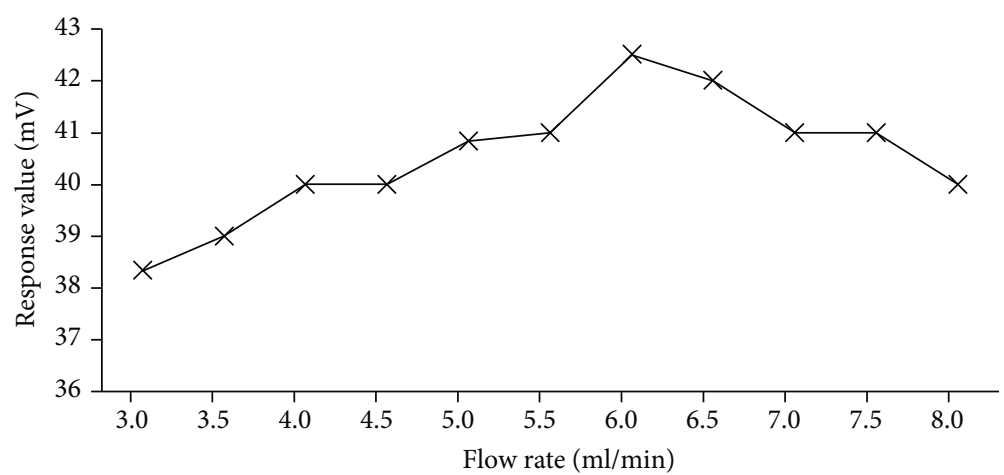

Figure 5: Effect of carrier gas flow rate on $200 \mathrm{ppb}$ toluene response value.

TABLE 1: Dimethyl sulfide and toluene separation results in different carrier gas flow rates.

\begin{tabular}{|c|c|c|c|c|}
\hline Flow rate $(\mathrm{ml} / \mathrm{min})$ & Substance category & Retention time (s) & Peak width & Separation degree \\
\hline \multirow{2}{*}{2} & Dimethyl sulfide & 78 & 26 & \multirow{2}{*}{2.79} \\
\hline & Toluene & 176 & 44 & \\
\hline \multirow{2}{*}{2.5} & Dimethyl sulfide & 65 & 37 & \multirow{2}{*}{2.13} \\
\hline & Toluene & 146 & 39 & \\
\hline \multirow{2}{*}{3} & Dimethyl sulfide & 55 & 46 & \multirow{2}{*}{1.66} \\
\hline & Toluene & 127 & 41 & \\
\hline \multirow{2}{*}{3.5} & Dimethyl sulfide & 47 & 46 & \multirow{2}{*}{1.51} \\
\hline & Toluene & 107 & 34 & \\
\hline \multirow{2}{*}{4} & Dimethyl sulfide & 44 & 33 & \multirow{2}{*}{1.68} \\
\hline & Toluene & 96 & 29 & \\
\hline \multirow{2}{*}{4.5} & Dimethyl sulfide & 37 & 33 & \multirow{2}{*}{1.53} \\
\hline & Toluene & 85 & 29 & \\
\hline \multirow{2}{*}{5} & Dimethyl sulfide & 34 & 26 & \multirow{2}{*}{1.68} \\
\hline & Toluene & 76 & 24 & \\
\hline \multirow{2}{*}{5.5} & Dimethyl sulfide & 28 & 20 & \multirow{2}{*}{1.63} \\
\hline & Toluene & 61 & 21 & \\
\hline \multirow{2}{*}{6} & Dimethyl sulfide & 26 & 26 & \multirow{2}{*}{1.50} \\
\hline & Toluene & 60 & 20 & \\
\hline \multirow{2}{*}{6.5} & Dimethyl sulfide & 24 & 20 & \multirow{2}{*}{1.74} \\
\hline & Toluene & 57 & 18 & \\
\hline \multirow[b]{2}{*}{7} & Dimethyl sulfide & 23 & 23 & \multirow{2}{*}{1.58} \\
\hline & Toluene & 54 & 16 & \\
\hline
\end{tabular}


efficiency increases, but at the same time, the mass transfer resistance term increases, which in turn reduces the column efficiency. The flow rate of the carrier gas influences the height of the tray, so there is an optimum flow rate so that the column efficiency is the highest. In this experiment, the sample gas was sampled using $200 \mathrm{ppb}$ toluene standard gas prepared by DaLian Date and the carrier gas used was $99.999 \%$ nitrogen $\left(\mathrm{N}_{2}\right)$. By changing the flow rate of the carrier gas before the column, the corresponding response value of the $200 \mathrm{ppb}$ toluene standard gas is shown in Figure 5. It shows that toluene response value had a peak value at about $6 \mathrm{ml} / \mathrm{min}$.

4.4. Effect of Carrier Gas Flow on Separation of Mixtures. A gas generator was used to prepare dimethyl sulfide and toluene gas mixture. The gas mixture was separated and detected by the gas chromatographic separation and detection system developed by our group $[10,11]$. In this experiment, $99.999 \%$ nitrogen $\left(\mathrm{N}_{2}\right)$ was used as the carrier gas. The temperature of the chip column is $85^{\circ} \mathrm{C}$, select the S48 300/HMT type mass flow controller manufactured, and control the flow rate at $2 \sim 7 \mathrm{ml} / \mathrm{min}$, and each flow rate is carried out. Two repeated injection tests were performed to determine the retention time and peak width of the mixture, and the mean value was calculated. Separation of the mixture is mainly achieved by using the separation of the system; the relative physical and chemical properties such as the boiling point, polarity, and adsorption and desorption of each component are fixed [12]. After separation and detection of dimethyl sulfide and toluene mixture, data processing was performed using the software of the upper computer to calculate the retention time and peak width of dimethyl sulfide and toluene at different flow rates, and the separation was calculated according to equation (3). The specific data results are shown in Table 1.

From Table 1, the flow rate is between 2 and $7 \mathrm{ml} / \mathrm{min}$, the separation degree of methyl sulfide and toluene is $R>$ 1.5 , and the two peaks are completely separated. The higher the degree of separation, the more accurate detection results could be [13]. When the flow rate is low, as the flow rate increases, the peak width of the peak narrows, the retention time becomes shorter, the number of theoretical plates becomes larger, and the column efficiency of the chip column increases [14].

\section{Conclusions}

During the rate theory, the flow rate of the carrier gas affects the column and column efficiency. Using the developed chiptype column at different carrier gas flow rates, experiments were carried out to examine the effect of carrier gas flow rate on the baseline value, $200 \mathrm{ppb}$ toluene response, and mixture separation. The flow rate was between 2 and $7 \mathrm{ml} / \mathrm{min}$, and the baseline value gradually decreased. The response value of $200 \mathrm{ppb}$ toluene reached maximum at a flow rate of about $6 \mathrm{ml} / \mathrm{min}$. The separation of dimethyl sulfide and toluene was not less than 1.5. Both were completely separated. Based on comprehensive column efficiency, gas response value, high separation of mixed gas, short analysis cycle, and other factors, the optimal carrier gas flow rate for the chip column is $6 \mathrm{ml} / \mathrm{min}$.

\section{Data Availability}

The data used to support the findings of this study are included within the article.

\section{Conflicts of Interest}

All the authors of this manuscript declare no competing financial interest.

\section{Acknowledgments}

This work is supported by the project of Tianjin University of Science and Technology (No. 2017KJ112): Development Fund Mechanism of laser-guided screening of circulating tumor cells.

\section{References}

[1] J. Pfahler, J. Harley, H. Bau, and J. Zemel, "Liquid transport in micro and submicron channels," Proceedings of SPIE, vol. 1167, no. 89, pp. 159-169, 1992.

[2] M. Yu, Z. Hongmei, Z. Hua, and Q. Dewen, "Research progress and application of microfluidic chip technology," Plant Protection, vol. 4, pp. 1-8, 2014.

[3] S. Dongping, Modern instrument analysis and experimental techniques (volume 1), Science Press, Beijing, 2015.

[4] L. Yang, Z. Sixiang, L. Shanshan, Z. Xu, Y. Xinying, and H. Xiaowei, "Development of the micro gas chromatography columns for malodorous gas detection," Micronanoelectronic Technology, vol. 7, pp. 447-450, 2014.

[5] L. Huwei, Gas chromatography method and its application, Chemical Industry Press, Beijing, 2007.

[6] Q. Meiling, Gas chromatographic analysis and application, vol. 43, Science Press, Beijing, 2012.

[7] H. Jintu, "Inquiry upon the separating rate of chromatography," Journal of Xinyang Teachers College (Natural Science Edition), vol. 4, pp. 351-353, 1990.

[8] M. Zhu and P. Hu, Instrumental analysis, Higher Education Press, Beijing, fourth edition, 2008.

[9] X. Zhang, L. Liu, Y. Xu, J. Li, F. Wang, and S. Zhang, "Odor gas online detection system and experiments research," Journal of Hebei University of Technology, vol. 3, pp. 38-43, 2015.

[10] X. Yang, The development and experimental research of odorous gases detecting system, Hebei University of Technology, Tianjin, 2015.

[11] F. Wang, "The research on data processing and calibration of odorous detection system," Hebei University of Technology, Tianjin, 2016.

[12] S. P. Cram and R. S. Juvet, "Gas chromatography," Analytical Chemistry, vol. 44, no. 5, pp. 213-241, 2002.

[13] Y. Haiming and S. Meihua, "Influence of resolution on quantitative analysis by gas chromatography," Pollution Control Technology, vol. 6, pp. 49-50, 2006.

[14] R. Nasreddine, V. Person, C. A. Serra, and S. le Calvé, "Development of a novel portable miniaturized GC for near real-time low level detection of BTEX," Sensors \& Actuators B Chemical, vol. 224, pp. 159-169, 2016. 